\title{
Laplacians with singular perturbations supported on hypersurfaces
}

\author{
A. Mantile ${ }^{1}$, A. Posilicano ${ }^{2}$ \\ ${ }^{1}$ Laboratoire de Mathématiques de Reims, EA4535 URCA, \\ Fédération de Recherche ARC Mathématiques, FR 3399 CNRS, France \\ ${ }^{2}$ DiSAT, Sezione di Matematica, Università dell'Insubria, via Valleggio 11, \\ I-22100 Como, Italy \\ andrea.mantile@univ-reims.fr, andrea.posilicano@uninsubria.it
}

PACS 02.30.Tb, 02.30.Jr

DOI 10.17586/2220-8054-2016-7-2-315-323

\begin{abstract}
We review the main results of our recent work on singular perturbations supported on bounded hypersurfaces. Our approach consists in using the theory of self-adjoint extensions of restrictions to build self-adjoint realizations of the $n$-dimensional Laplacian with linear boundary conditions on (a relatively open part of) a compact hypersurface. This allows one to obtain Krein-like resolvent formulae where the reference operator coincides with the free selfadjoint Laplacian in $\mathbb{R}^{n}$, providing in this way with an useful tool for the scattering problem from a hypersurface. As examples of this construction, we consider the cases of Dirichlet and Neumann boundary conditions assigned on an unclosed hypersurface.
\end{abstract}

Keywords: Kreĭn's resolvent formula, boundary conditions, self-adjoint extensions.

Received: 02 March 2016

\section{Introduction}

In a recent paper [1], the complete family of self-adjoint elliptic operators with interface conditions assigned on a hypersurface in $\mathbb{R}^{n}$ was realized. Derived from the abstract theory of selfadjoint extensions of restrictions developed in [2-5], our approach leads to Kreĭn type formulae for the resolvent difference between the perturbed operator and the corresponding free selfadjoint model with domain $H^{2}\left(\mathbb{R}^{n}\right)$. This is a relevant point for the interface perspective of studying the scattering problem. Moreover, while some sub-families of extensions (mainly those concerned with the $\delta$ or $\delta^{\prime}$ interface conditions) have been largely investigated by using quadratic form or quasi-boundary triple techniques (see [6-25]), for others models presented in [1], and in particular those concerned with local interface conditions of Dirichlet and Neumann type, a rigorous analysis was not previously given.

The aim of this report is to provide a shortened introduction to this analysis, giving the essential information about the construction of our models in the case of singular perturbations of the $n$-dimensional Laplacian with interface conditions. In this framework, we recall the basic results needed to construct the whole family of singular perturbations and then focus on the explicit examples of "global" and "local" Dirichlet- and Neumann-type boundary conditions. For the detailed proofs, we refer to [1].

After recalling in Section 1 the main properties of the trace maps and the layer operators related to the surface $\Gamma$, we introduce our model in Section 2 through the symmetric operator:

$$
\Delta^{\circ}=\Delta \uparrow\left\{u \in H^{2}\left(\mathbb{R}^{n}\right): u|\Gamma=(\nu \cdot \nabla) u| \Gamma=0\right\},
$$

where $\nu$ denotes the exterior unit normal on $\Gamma$. The self-adjoint realizations of the Laplacian with boundary conditions involving linear relations between lateral traces on $\Gamma$, or on a relatively open 
part $\Sigma \subset \Gamma$, are defined as selfadjoint extensions of $\Delta^{\circ}$. The general construction provided in [2-5] allows us to define these extensions as singular perturbations of the free Laplacian operator defined by $\operatorname{dom}(\Delta)=H^{2}\left(\mathbb{R}^{n}\right)$. In this framework, the perturbed operators are parametrized through couples $(\Pi, \Theta)$, where $\Pi$ is an orthogonal projector on the Hilbert trace space $H^{3 / 2}(\Gamma) \oplus$ $H^{1 / 2}(\Gamma)$ and $\Theta$ is a self-adjoint operator in the Hilbert space given by the range of $\Pi$. In Theorem 3.1 and Corollary 3.5, we define this family of extensions in terms $(\Pi, \Theta)$ and give the corresponding Krein-like resolvent formulae, while their spectral properties and the conditions for the wave operators existence and completeness are given in Theorem 3.3. The connection between this abstract parametrization and explicit boundary (or interface) conditions is the main issue concerned with this approach. In Section 3 we consider this point in the particular cases of the Dirichlet and Neumann conditions on $\Gamma$ and on $\Sigma \subset \Gamma$.

\section{Preliminaries}

Let $\Omega \subset \mathbb{R}^{n}$ be open and suppose its boundary $\Gamma=\partial \Omega$ is a smooth $(n-1)$-dimensional compact manifold. In this case, $H^{s}(\Omega), s \in \mathbb{R}$, is defined by $H^{s}(\Omega):=\left\{u \mid \Omega: u \in H^{s}\left(\mathbb{R}^{n}\right)\right\}$, $u \mid \Omega$ denoting the restriction of $u$ to $\Omega$ and $H^{s}\left(\mathbb{R}^{n}\right)$ denoting the usual scale of Hilbert-Sobolev spaces on $\mathbb{R}^{n}$ defined by Fourier transform. The Sobolev spaces of $L^{2}$-functions on $\Gamma$, next denoted with $H^{s}(\Gamma)$, are defined by using an atlas of $\Gamma$ and the Sobolev space on flat, open, bounded, $(n-1)$-dimensional domains (see e.g. [26, Chapter 1], [27, Chapter 3]); $H^{-s}(\Gamma)$ identifies with the dual space of $H^{s}(\Gamma) ;\langle\cdot, \cdot\rangle_{-s, s}$ denotes the $H^{-s}-H^{s}$ duality. Considering the Riemannian structure inherited from $\mathbb{R}^{n}$, the space $H^{s}(\Gamma)$ identifies with $\operatorname{dom}\left(\left(-\Delta_{\Gamma}\right)^{s / 2}\right)$ with respect to the scalar product:

$$
\langle\phi, \varphi\rangle_{H^{s}(\Gamma)}:=\left\langle\Lambda^{s} \phi, \Lambda^{s} \varphi\right\rangle_{L^{2}(\Gamma)}, \quad \Lambda:=\left(-\Delta_{\Gamma}+1\right)^{1 / 2},
$$

being $\Delta_{\Gamma}$ the self-adjoint operator in $L^{2}(\Gamma)$ corresponding to the Laplace-Beltrami operator on the complete Riemannian manifold $\Gamma$ (see e.g. [26, Remark 7.6, Chapter 1]). According to this definition, $\Lambda^{r}$ is self-adjoint in $H^{s}(\Gamma)$ with domain $H^{s+r}(\Gamma)$ and acts as a unitary map $\Lambda^{r}: H^{s}(\Gamma) \rightarrow H^{s-r}(\Gamma)$.

For a bounded open domain $\Omega$, we set: $\Omega_{-}=\Omega$ and $\Omega_{+}=\mathbb{R}^{n} \backslash \Omega$, while $\nu$ denotes the outward normal vector on $\Gamma=\partial \Omega$. The domain of the maximal Laplacian in $L^{2}\left(\Omega_{ \pm}\right)$is next denoted by:

$$
L_{\Delta}^{2}\left(\Omega_{ \pm}\right):=\left\{u \in L^{2}(\Omega): \Delta u \in L^{2}\left(\Omega_{ \pm}\right)\right\}
$$

and we define:

$$
L_{\Delta}^{2}\left(\mathbb{R}^{n} \backslash \Gamma\right):=L_{\Delta}^{2}\left(\Omega_{-}\right) \oplus L_{\Delta}^{2}\left(\Omega_{+}\right)
$$

We also pose:

$$
H^{s}\left(\mathbb{R}^{n} \backslash \Gamma\right):=H^{s}\left(\Omega_{-}\right) \oplus H^{s}\left(\Omega_{+}\right) .
$$

The one-sided, zero-order, trace operators $\gamma_{0}^{ \pm}$act on a smooth function $u \in \mathcal{C}^{\infty}\left(\bar{\Omega}_{ \pm}\right)$as $\gamma_{0}^{ \pm} u=u \mid \Gamma$, where $\varphi \mid \Gamma$ is the restriction to $\Gamma$. These maps uniquely extend to bounded linear operators (see e.g. [27, Theorem 3.37]):

$$
\gamma_{0}^{ \pm} \in \mathrm{B}\left(H^{s}\left(\Omega_{ \pm}\right), H^{s-1 / 2}(\Gamma)\right), \quad s>\frac{1}{2} .
$$

The one-sided first-order trace operators are given by $\gamma_{1}^{ \pm} u:=\nu \cdot \gamma_{0}^{ \pm}(\nabla u)$; from (6) there follows:

$$
\gamma_{1}^{ \pm} \in \mathrm{B}\left(H^{s}\left(\Omega_{ \pm}\right), H^{s-\frac{3}{2}}(\Gamma)\right), \quad s>\frac{3}{2} .
$$


Using these maps, the two-sided bounded trace operators are defined according to:

$$
\begin{aligned}
& \gamma_{0}: H^{s}\left(\mathbb{R}^{n} \backslash \Gamma\right) \rightarrow H^{s-\frac{1}{2}}(\Gamma), \quad \gamma_{0}\left(u_{-} \oplus u_{+}\right):=\frac{1}{2}\left(\gamma_{0}^{+} u_{+}+\gamma_{0}^{-} u_{-}\right), \\
& \gamma_{1}: H^{s}\left(\mathbb{R}^{n} \backslash \Gamma\right) \rightarrow H^{s-\frac{3}{2}}(\Gamma), \quad \gamma_{1}\left(u_{-} \oplus u_{+}\right):=\frac{1}{2}\left(\gamma_{1}^{+} u_{+}+\gamma_{1}^{-} u_{-}\right),
\end{aligned}
$$

while the corresponding jumps are:

$$
\begin{array}{ll}
{\left[\gamma_{0}\right]: H^{s}\left(\mathbb{R}^{n} \backslash \Gamma\right) \rightarrow H^{s-\frac{1}{2}}(\Gamma),} & {\left[\gamma_{0}\right]\left(u_{-} \oplus u_{+}\right):=\gamma_{0}^{+} u_{+}-\gamma_{0}^{-} u_{-},} \\
{\left[\gamma_{1}\right]: H^{s}\left(\mathbb{R}^{n} \backslash \Gamma\right) \rightarrow H^{s-\frac{3}{2}}(\Gamma),} & {\left[\gamma_{1}\right]\left(u_{-} \oplus u_{+}\right):=\gamma_{1}^{+} u_{+}-\gamma_{1}^{-} u_{-} .}
\end{array}
$$

By [26, Theorem 6.5, Section 6, Chapter 2], the maps $\gamma_{0}^{ \pm}$and $\gamma_{1}^{ \pm}$can be further extended to:

$$
\hat{\gamma}_{0}^{ \pm} \in \mathrm{B}\left(L_{\Delta}^{2}\left(\Omega_{ \pm}\right), H^{-1 / 2}(\Gamma)\right)
$$

and

thus producing the extended jumps maps:

$$
\hat{\gamma}_{1}^{ \pm} \in \mathrm{B}\left(L_{\Delta}^{2}\left(\Omega_{ \pm}\right), H^{-\frac{3}{2}}(\Gamma)\right)
$$

$$
\begin{array}{ll}
{\left[\hat{\gamma}_{0}\right]: L_{\Delta}^{2}\left(\mathbb{R}^{n} \backslash \Gamma\right) \rightarrow H^{-\frac{1}{2}}(\Gamma),} & {\left[\hat{\gamma}_{0}\right]\left(u_{-} \oplus u_{+}\right):=\hat{\gamma}_{0}^{+} u_{+}-\hat{\gamma}_{0}^{-} u_{-},} \\
{\left[\hat{\gamma}_{1}\right]: L_{\Delta}^{2}\left(\mathbb{R}^{n} \backslash \Gamma\right) \rightarrow H^{-\frac{3}{2}}(\Gamma), \quad\left[\hat{\gamma}_{1}\right]\left(u_{-} \oplus u_{+}\right):=\hat{\gamma}_{1}^{+} u_{+}-\hat{\gamma}_{1}^{-} u_{-} .}
\end{array}
$$

In what follows, the $n$-dimensional free Laplacian is defined by $\operatorname{dom}(\Delta)=H^{2}\left(\mathbb{R}^{n}\right)$. This is a selfadjoint and negatively-defined operator with: $\sigma(\Delta)=\sigma_{a c}(\Delta)=(-\infty, 0]$, and for all $z \in \mathbb{C} \backslash \mathbb{R}_{-}$it follows that:

$$
(-\Delta+z)^{-1} \in \mathrm{B}\left(H^{s}\left(\mathbb{R}^{n}\right), H^{s+2}\left(\mathbb{R}^{n}\right)\right)
$$

Given an open and bounded smooth domain $\Omega$, the single and double-layer operators related to $(-\Delta+z)^{-1}$ and to the surface $\Gamma=\partial \Omega$ are defined for any $z \in \mathbb{C} \backslash \mathbb{R}$ - by:

$$
\begin{aligned}
& \left\langle S L_{z} \phi, u\right\rangle_{L^{2}\left(\mathbb{R}^{n}\right)}:=\left\langle\phi, \gamma_{0}(-\Delta+\bar{z})^{-1} u\right\rangle_{-3 / 2,3 / 2}, \\
& \left\langle D L_{z} \varphi, u\right\rangle_{L^{2}\left(\mathbb{R}^{n}\right)}:=\left\langle\varphi, \gamma_{1}(-\Delta+\bar{z})^{-1} u\right\rangle_{-1 / 2,1 / 2} .
\end{aligned}
$$

Due to the mapping properties (6) - (7) and (16), these relation define bounded maps on $H^{-3 / 2}(\Gamma)$ and $H^{-1 / 2}(\Gamma)$, provided that $z \in \mathbb{C} \backslash \mathbb{R}_{-}$; we have:

$$
S L_{z} \in \mathrm{B}\left(H^{-3 / 2}(\Gamma), L^{2}\left(\mathbb{R}^{n}\right)\right), \quad D L_{z} \in \mathrm{B}\left(H^{-1 / 2}(\Gamma), L^{2}\left(\mathbb{R}^{n}\right)\right) .
$$

The integral kernel of $(-\Delta+z)^{-1}, z \in \mathbb{C} \backslash \mathbb{R}_{-}$, is given by:

$$
\mathcal{K}_{z}(x-y)=\frac{1}{2 \pi}\left(\frac{\sqrt{z}}{2 \pi\|x-y\|}\right)^{n / 2-1} K_{n / 2-1}(\sqrt{z}\|x-y\|), \quad \operatorname{Re} \sqrt{z}>0
$$

where $K_{\alpha}$ denotes the modified Bessel functions of second kind of order $\alpha$. This is a smooth function for $x \neq y$ and the relations (17) and (18) give:

$$
S L_{z} \phi(x)=\int_{\Gamma} \mathcal{K}_{z}(x-y) \phi(y) d \sigma_{\Gamma}(y), \quad x \notin \Gamma \text { and } \phi \in L^{2}(\Gamma),
$$

and

$$
D L_{z} \varphi(x)=\int_{\Gamma} \nu(y) \cdot \nabla \mathcal{K}_{z}(x-y) \varphi(y) d \sigma_{\Gamma}(y), \quad x \notin \Gamma \text { and } \varphi \in L^{2}(\Gamma),
$$

where $\sigma_{\Gamma}$ denotes the surface measure. In particular, one has (see [27, eqs. (6.18) and (6.19)]):

$$
\forall x \notin \Gamma, \quad \Delta S L_{z} \phi(x)=z S L_{z} \phi(x), \quad \Delta D L_{z} \varphi(x)=z D L_{z} \varphi(x),
$$


from which, we obtain

$$
S L_{z} \in \mathrm{B}\left(H^{-3 / 2}(\Gamma), L_{\Delta}^{2}\left(\mathbb{R}^{n} \backslash \Gamma\right)\right), \quad D L_{z} \in \mathrm{B}\left(H^{-1 / 2}\left(\mathbb{R}^{n} \backslash \Gamma\right), L_{\Delta}^{2}\left(\mathbb{R}^{n} \backslash \Gamma\right)\right) ;
$$

in particular, the representation:

$$
L_{\Delta}^{2}\left(\mathbb{R}^{n} \backslash \Gamma\right)=\left\{u=u_{0}+S L_{z} \phi+D L_{z} \varphi, u_{0} \in H^{2}\left(\mathbb{R}^{n}\right), \phi \oplus \varphi \in H^{-3 / 2}(\Gamma) \oplus H^{-1 / 2}(\Gamma)\right\},
$$

holds for any $z \in \mathbb{C} \backslash \mathbb{R}_{-}$(see [1, Lemma 4.2]). In the following, we choose $z=1$ and set

$$
S L:=S L_{1}, \quad D L:=D L_{1} \text {. }
$$

\section{Singular perturbations supported on hypersurfaces.}

Let $\Omega \subset \mathbb{R}^{n}$, be open and bounded with smooth boundary $\Gamma$ and denote:

$$
\gamma: H^{2}\left(\mathbb{R}^{n}\right) \rightarrow H^{3 / 2}(\Gamma) \oplus H^{1 / 2}(\Gamma), \quad \gamma u:=\gamma_{0} u \oplus \gamma_{1} u .
$$

The singular perturbations of the free Laplacian supported on $\Gamma$ are next defined as the selfadjoint extensions of the closed symmetric operator:

$$
\Delta^{\circ}:=\Delta \uparrow \operatorname{ker}(\gamma)
$$

where

$$
\operatorname{ker}(\gamma)=H_{0}^{2}\left(\Omega_{+}\right) \oplus H_{0}^{2}\left(\Omega_{-}\right), \quad H_{0}^{2}\left(\Omega_{ \pm}\right):=\left\{u_{ \pm} \in H^{2}\left(\Omega_{ \pm}\right): \gamma_{0}^{ \pm} u_{ \pm}=\gamma_{1}^{ \pm} u_{ \pm}=0\right\}
$$

The corresponding adjoint coincides with the maximal Laplacian in $\mathbb{R}^{n} \backslash \Gamma$, i.e.

$$
\left(\Delta^{\circ}\right)^{*}=\Delta \uparrow L_{\Delta}^{2}\left(\Omega_{+}\right) \oplus L_{\Delta}^{2}\left(\Omega_{-}\right) \text {. }
$$

Using the alternative representation given in (24), we have:

$$
\left(\Delta^{\circ}\right)^{*} u=\Delta u_{0}+z\left(S L_{z} \phi+D L_{z} \varphi\right) .
$$

Moreover, $\left(\Delta^{\circ}\right)^{*}$ and the distributional Laplacian are related by the identity (see e.g. in [28, Theorem 3.1]):

$$
\left(\Delta^{\circ}\right)^{*} u=\Delta u-\left[\hat{\gamma}_{1}\right] u \delta_{\Gamma}-\left[\hat{\gamma}_{0}\right] u \partial_{\nu} \delta_{\Gamma}
$$

Here, for $f \in H^{-s}(\Gamma), f \delta_{\Gamma}$ and $f \partial_{\nu} \delta_{\Gamma}$ are the distributions supported on $\Gamma$ defined by:

$$
\left(f \delta_{\Gamma}, \chi\right)=\left\langle\bar{f}, \gamma_{0} \chi\right\rangle_{-s, s}, \text { and }\left(f \partial_{\nu} \delta_{\Gamma}, \chi\right)=-\left\langle\bar{f}, \gamma_{1} \chi\right\rangle_{-s, s} .
$$

In particular, taking $f=1$, for any $\chi \in \mathcal{C}_{0}^{\infty}(\Omega)$ one has:

$$
\left(\delta_{\Gamma}, \chi\right)=\int_{\Gamma} \chi(x) d \sigma_{\Gamma}(x)
$$

and

$$
\left(\partial_{\nu} \delta_{\Gamma}, \chi\right)=-\int_{\Gamma} \partial_{\nu} \chi(x) d \sigma_{\Gamma}(x)
$$

Let us recall that $\gamma$ belongs to $\mathrm{B}\left(H^{2}\left(\mathbb{R}^{n}\right), H^{3 / 2}(\Gamma) \oplus H^{1 / 2}(\Gamma)\right)$, is surjective and has a kernel dense in $L^{2}\left(\mathbb{R}^{n}\right)$ [1, Lemma 4.1]. Hence, the approach developed in [2-5] applies to our framework and allows us to construct all self-adjoint extensions of $\Delta^{\circ}$. For generic elliptic selfadjoint operators with smooth coefficients, this strategy has been implemented in [1] to which we refer for the detailed proofs. The auxiliary operators $G_{z}$ are next defined by the duality:

$$
\left\langle G_{z} \xi, u\right\rangle_{L^{2}\left(\mathbb{R}^{n}\right)}=\left\langle\xi, \gamma(-\Delta+z)^{-1} u\right\rangle_{\Gamma}, \quad \xi \in H^{-3 / 2}(\Gamma) \oplus H^{-1 / 2}(\Gamma), u \in L^{2}\left(\mathbb{R}^{n}\right) .
$$

for all $z \in \mathbb{C} \backslash \mathbb{R}_{-}$. From (17) - (18) it easily follows that:

$$
G_{z} \in \mathrm{B}\left(H^{-3 / 2}(\Gamma) \oplus H^{-1 / 2}(\Gamma), L^{2}\left(\mathbb{R}^{n}\right)\right), \quad G_{z}(\phi \oplus \varphi)=S L_{z} \phi+D L_{z} \varphi .
$$


In what follows, we set:

$$
G:=G_{1}, \quad G(\phi \oplus \varphi)=S L \phi+D L \varphi .
$$

With this notation, the adjoint $\left(\Delta^{\circ}\right)^{*}$ is rephrased as:

$$
\begin{gathered}
\operatorname{dom}\left(\left(\Delta^{\circ}\right)^{*}\right)=\left\{u=u_{0}+G(\phi \oplus \varphi), u_{0} \in H^{2}\left(\mathbb{R}^{n}\right), \phi \oplus \varphi \in H^{-3 / 2}(\Gamma) \oplus H^{-1 / 2}(\Gamma)\right\}, \\
\left(\Delta_{0}\right)^{*}=\Delta u_{0}+G(\phi \oplus \varphi) .
\end{gathered}
$$

We introduce the map: $M_{z}=\gamma\left(G-G_{z}\right)$ whose action on $H^{-3 / 2}(\Gamma) \oplus H^{-1 / 2}(\Gamma)$ is explicitly given by:

$$
M_{z}:=\left(\begin{array}{ll}
\gamma_{0}\left(S L-S L_{z}\right) & \gamma_{0}\left(D L-D L_{z}\right) \\
\gamma_{1}\left(S L-S L_{z}\right) & \gamma_{1}\left(D L-D L_{z}\right)
\end{array}\right)
$$

From [1, eq. (2.6)], it results that:

$$
M_{z} \in \mathrm{B}\left(H^{-3 / 2}(\Gamma) \oplus H^{-1 / 2}(\Gamma), H^{3 / 2}(\Gamma) \oplus H^{1 / 2}(\Gamma)\right) .
$$

In what follows,

$$
\Pi: H^{3 / 2}(\Gamma) \oplus H^{1 / 2}(\Gamma) \rightarrow H^{3 / 2}(\Gamma) \oplus H^{1 / 2}(\Gamma)
$$

denotes an orthogonal projector on the Hilbert space $H^{3 / 2}(\Gamma) \oplus H^{1 / 2}(\Gamma)$,

$$
\Pi^{\prime}: H^{-3 / 2}(\Gamma) \oplus H^{-1 / 2}(\Gamma) \rightarrow H^{-3 / 2}(\Gamma) \oplus H^{-1 / 2}(\Gamma),
$$

is the corresponding dual projector and

$$
\Theta: \operatorname{dom}(\Theta) \subseteq \operatorname{ran}(\Pi)^{\prime} \rightarrow \operatorname{ran}(\Pi)
$$

is selfadjoint in the sense of the duality, i.e.: $\Theta=\Theta^{\prime}$. In this framework, the selfadjoint extensions of $\Delta^{\circ}$ are parametrized by the couples $(\Pi, \Theta)$. In particular, adapting [1, Theorem 2.1 and Lemma 4.9] to the present framework, there follows:

Theorem 3.1. Let $\Pi: H^{3 / 2}(\Gamma) \oplus H^{1 / 2}(\Gamma) \rightarrow H^{3 / 2}(\Gamma) \oplus H^{1 / 2}(\Gamma)$ be an orthogonal projector and $\Theta: \operatorname{dom}(\Theta) \subseteq \operatorname{ran}(\Pi)^{\prime} \rightarrow \operatorname{ran}(\Pi)$ selfadjoint. Any self-adjoint extension of $\Delta_{0}$ is of the kind $\Delta_{\Pi, \Theta}$,

$$
\begin{gathered}
\Delta_{\Pi, \Theta}:= \\
\left(\Delta^{\circ}\right)^{*} \uparrow\left\{u=u_{0}+S L \phi+D L \varphi, u_{0} \in H^{2}\left(\mathbb{R}^{n}\right), \phi \oplus \varphi \in \operatorname{dom}(\Theta): \Pi \gamma u_{0}=\Theta(\phi \oplus \varphi)\right\} .
\end{gathered}
$$

The set:

$$
Z_{\Pi, \Theta}:=\left\{z \in \mathbb{C} \backslash \mathbb{R}_{-}: \Theta+\Pi M_{z} \Pi^{\prime} \text { has a bounded inverse }\right\},
$$

is not void; in particular, $\mathbb{C} \backslash \mathbb{R} \subseteq Z_{\Pi, \Theta} \subseteq \operatorname{res}\left(\Delta_{\Pi, \Theta}\right)$ and for any $z \in Z_{\Pi, \Theta}$ the resolvent of $\Delta_{\Pi, \Theta}$ is given by the Krěin type formula:

$$
\left(-\Delta_{\Pi, \Theta}+z\right)^{-1} u=(-\Delta+z)^{-1} u+G_{z} \Pi^{\prime}\left(\Theta+\Pi M_{z} \Pi^{\prime}\right)^{-1} \Pi \gamma(-\Delta+z)^{-1} u,
$$

where $G_{z}$ and $M_{z}$ are defined in (36) and (40) respectively.

Remark 3.2. Let us notice that the $\Pi^{\prime}$ appearing in (47) act as the inclusion map $\Pi^{\prime}: \operatorname{ran}(\Pi)^{\prime} \rightarrow$ $H^{-3 / 2}(\Gamma) \oplus H^{-1 / 2}(\Gamma)$. This means that one does not need to know $\Pi^{\prime}$ explicitly: it suffices to know the subspace $\operatorname{ran}\left(\Pi^{\prime}\right)=\operatorname{ran}(\Pi)^{\prime}$.

The next result gives information on the spectrum and scattering of $\Delta_{\Pi, \Theta}$. For the proof of such results, we refer to [1, Lemma 4.9, Corollary 4.12 and Remark 4.14]. Let us remark that hypothesis (49) below typically holds in the case of global boundary conditions, i.e. assigned on whole boundary $\Gamma$, while hypothesis (50) typically holds in the case of local ones, i.e. assigned on an open part $\Sigma \subset \Gamma$. 
Theorem 3.3. 1) Suppose:

Then,

$$
\operatorname{dom}(\Theta) \subseteq H^{s_{1}}(\Gamma) \oplus H^{s_{2}}(\Gamma), \quad s_{1}>-\frac{3}{2}, \quad s_{2}>-\frac{1}{2} .
$$

2) Suppose either:

$$
\sigma_{e s s}\left(\Delta_{\Pi, \Theta}\right)=(-\infty, 0]
$$

$$
\operatorname{dom}(\Theta) \subseteq H^{\frac{1}{2}}(\Gamma) \oplus H^{\frac{3}{2}}(\Gamma)
$$

or

$$
\operatorname{dom}\left(f_{\tilde{\Theta}}\right) \subseteq H^{5 / 2}(\Gamma) \oplus H^{\frac{3}{2}}(\Gamma),
$$

holds, where $f_{\tilde{\Theta}}$ is sesquilinear form associated to the self-adjoint operator in $\operatorname{ran}(\Pi)$ defined by $\tilde{\Theta}:=\Theta\left(\Lambda^{3} \oplus \Lambda\right)$. Then:

and the wave operators:

$$
\sigma_{a c}\left(\Delta_{\Pi, \Theta}\right)=(-\infty, 0]
$$

$$
W_{ \pm}:=s^{-} \lim _{t \rightarrow \pm \infty} e^{-i t \Delta_{\Pi, \Theta}} e^{i t \Delta}, \quad W_{ \pm}:=s^{-} \lim _{t \rightarrow \pm \infty} e^{-i t \Delta} e^{i t \Delta_{\Pi, \Theta}} P_{a c}
$$

exist and are complete, i.e. the limits exists everywhere w.r.t. strong convergence, $\operatorname{ran}\left(W_{ \pm}\right)=$ $L^{2}\left(\mathbb{R}^{n}\right)_{a c}, \operatorname{ran}\left(W_{ \pm}\right)=L^{2}\left(\mathbb{R}^{n}\right)$ and $W_{ \pm}^{*}=W_{ \pm}$, where $L^{2}\left(\mathbb{R}^{n}\right)_{a c}$ denotes the absolutely continuous subspace of $L^{2}\left(\mathbb{R}^{n}\right)$ with respect to $\Delta_{\Pi, \Theta}$ and $P_{a c}$ is the corresponding orthogonal projector.

Remark 3.4. Let us notice that the apparent discrepancy between the indices in the two conditions (49) and (50) is due to the fact that the first one applies to operators acting between the dual pair $\left(\operatorname{ran}(\Pi)^{\prime}, \operatorname{ran}(\Pi)\right)$, whereas the second one regards sesquilinear forms in the space $\operatorname{ran}(\Pi)$. When written in terms of $\tilde{\Theta}$, condition (49) reads as $\operatorname{dom}(\tilde{\Theta}) \subseteq H^{\frac{7}{2}}(\Gamma) \oplus H^{\frac{5}{2}}(\Gamma)$.

Under hypothesis (49), it is possible to introduce an alternative description of $\Delta_{\Pi, \Theta}$ (see [1, Corollary 4.8]):

Corollary 3.5. Let $\Delta_{\Pi, \Theta}$ be defined according to Theorem 3.1 with $\Theta$ fulfilling (49). Define

$$
B_{\Theta}:=\Theta+\Pi \gamma G \Pi^{\prime}: \operatorname{dom}(\Theta) \subseteq \operatorname{ran}\left(\Pi^{\prime}\right) \rightarrow \operatorname{ran}(\Pi) .
$$

Then:

$$
\operatorname{dom}\left(\Delta_{\Pi, \Theta}\right)=\left\{u \in H^{2}\left(\mathbb{R}^{n} \backslash \Gamma\right):[\gamma] u \in \operatorname{dom}(\Theta), \Pi \gamma u=B_{\Theta}[\gamma] u\right\}
$$

where $[\gamma] u:=\left(-\left[\gamma_{1}\right] u\right) \oplus\left(\left[\gamma_{0}\right] u\right)$, and

$$
\left(-\Delta_{\Pi, \Theta}+z\right)^{-1}-(-\Delta+z)^{-1}=G_{z} \Pi^{\prime}\left(B_{\Theta}-\Pi \gamma G_{z} \Pi^{\prime}\right)^{-1} \Pi \gamma(-\Delta+z)^{-1}, \quad z \in Z_{\Pi, \Theta} .
$$

\section{Dirichlet and Neumann boundary conditions on $\Sigma \subseteq \Gamma$}

In this section, we apply our results to self-adjoint adjoint realizations of the Laplacian with Dirichlet and Neumann type boundary conditions on $\Sigma \subseteq \Gamma$. For proofs and more details on such realizations, we refer to [1, Sections 5-6]. In particular, by the results given there, hypothesis (49) or (50) hold for the models considered here, namely: (49) is satisfied in the case of "global" boundary conditions (i.e. assigned on the whole $\Gamma$ ), while (50) holds in the case of "local" boundary conditions (i.e. assigned on $\Sigma \subset \Gamma$ ).

In the following, given $X \subset \Gamma$ closed, we use the definition:

$$
H_{X}^{s}(\Gamma):=\left\{\phi \in H^{s}(\Gamma): \operatorname{supp}(\phi) \subseteq X\right\}
$$

Given $\Sigma \subset \Gamma$ relatively open with a Lipschitz boundary, we denote by $\Pi_{\Sigma}$ the orthogonal projector in the Hilbert space $H^{s}(\Gamma), s>0$, such that $\operatorname{ran}\left(\Pi_{\Sigma}\right)=H_{\Sigma^{c}}^{s}(\Gamma)^{\perp}$. One has ran $\left(\Pi_{\Sigma}^{\prime}\right)=$ 
$H_{\bar{\Sigma}}^{-s}(\Gamma)$. Moreover, we use the identifications $H_{\Sigma^{c}}^{s}(\Gamma)^{\perp} \simeq H^{s}(\Sigma)$ and $H_{\bar{\Sigma}}^{-s}(\Gamma) \simeq H^{s}(\Sigma)^{\prime}$. In particular, by the former, the orthogonal projection $\Pi_{\Sigma}$ can be identified with the restriction map $R_{\Sigma}: H^{s}(\Gamma) \rightarrow H^{s}(\Sigma), R_{\Sigma} \phi:=\phi \mid \Sigma$.

\subsection{Dirichlet boundary conditions}

The self-adjoint extension $\Delta_{D}$ corresponding to Dirichlet boundary conditions on the whole $\Gamma$ is given by the direct sum $\Delta_{D}=\Delta_{\Omega_{+}}^{D} \oplus \Delta_{\Omega_{-}}^{D}$, where

$$
\Delta_{\Omega_{ \pm}}^{D}:=\Delta \uparrow\left\{u \in H^{2}\left(\Omega_{ \pm}\right): \gamma_{0}^{ \pm} u=0\right\}
$$

Since:

$$
\operatorname{dom}\left(\Delta_{\Omega_{+}}^{D}\right) \oplus \operatorname{dom}\left(\Delta_{\Omega_{-}}^{D}\right)=\left\{u \in H^{2}\left(\mathbb{R}^{n} \backslash \Gamma\right):\left[\gamma_{0}\right] u=0, \gamma_{0} u=0\right\}
$$

with the parametrization introduced in Corollary 3.5, this corresponds to the choice $\Pi(\phi \oplus \varphi):=$ $\phi \oplus 0$, and $B_{\Theta}=0$. Hence, from (31) we get:

$$
\Delta_{D} u=\Delta u-\left[\gamma_{1}\right] u \delta_{\Gamma} .
$$

Moreover, using the identity: $\left(\gamma_{0} S L_{z}\right)^{-1}=P_{z}^{-}-P_{z}^{+}$, where $P_{z}^{ \pm}$denote the Dirichlet-toNeumann operators for $\Omega_{ \pm}$respectively (see e.g. [1, equation (5.4)]), one has, for any $z \in \mathbb{C} \backslash \mathbb{R}_{-}$,

$$
\left(-\Delta_{D}+z\right)^{-1}=(-\Delta+z)^{-1}+S L_{z}\left(P_{z}^{+}-P_{z}^{-}\right) \gamma_{0}(-\Delta+z)^{-1} .
$$

Now, we turn to Dirichlet boundary conditions supported on a relatively open part $\Sigma \subset \Gamma$ with Lipschitz boundary. We denote by $\Delta_{D, \Sigma}$ the self-adjoint extension corresponding to the orthogonal projector defined by $\Pi(\phi \oplus \varphi):=\left(\Pi_{\Sigma} \phi\right) \oplus 0 \equiv(\phi \mid \Sigma) \oplus 0$ and to the self-adjoint operator $\Theta(\phi \oplus \varphi):=\left(-\Theta_{D, \Sigma} \phi\right) \oplus 0$ :

$$
\begin{gathered}
\Theta_{D, \Sigma}: \operatorname{dom}\left(\Theta_{D, \Sigma}\right) \subseteq H_{\bar{\Sigma}}^{-3 / 2}(\Gamma) \rightarrow H^{3 / 2}(\Sigma), \quad \Theta_{D, \Sigma} \phi:=\left(\gamma_{0} S L \phi\right) \mid \Sigma, \\
\operatorname{dom}\left(\Theta_{D, \Sigma}\right):=\left\{\phi \in H_{\bar{\Sigma}}^{-1 / 2}(\Gamma):\left(\gamma_{0} S L \phi\right) \mid \Sigma \in H^{3 / 2}(\Sigma)\right\} .
\end{gathered}
$$

By Theorem 3.1 and (31), one has:

$$
\begin{gathered}
\Delta_{D, \Sigma} u=\Delta u-\left[\hat{\gamma}_{1}\right] u \delta_{\bar{\Sigma}}, \\
\operatorname{dom}\left(\Delta_{D, \Sigma}\right)=\left\{u \in H^{1}\left(\mathbb{R}^{n}\right) \cap L_{\Delta}^{2}\left(\mathbb{R}^{n} \backslash \Gamma\right):\left[\hat{\gamma}_{1}\right] u \in \operatorname{dom}\left(\Theta_{D, \Sigma}\right),\left(\gamma_{0} u\right) \mid \Sigma=0\right\}, \\
\subseteq\left\{u \in H^{1}\left(\mathbb{R}^{n}\right) \cap L_{\Delta}^{2}\left(\mathbb{R}^{n} \backslash \Gamma\right):\left(\gamma_{0}^{-} u\right)\left|\Sigma=\left(\gamma_{0}^{+} u\right)\right| \Sigma=0,\left(\left[\hat{\gamma}_{1}\right] u\right) \mid \bar{\Sigma}^{c}=0\right\}
\end{gathered}
$$

and

$$
\left(-\Delta_{D, \Sigma}+z\right)^{-1}=(-\Delta+z)^{-1}-S L_{z} \Pi_{\Sigma}^{\prime}\left(R_{\Sigma} \gamma_{0} S L_{z} \Pi_{\Sigma}^{\prime}\right)^{-1} R_{\Sigma} \gamma_{0}(-\Delta+z)^{-1} .
$$

\subsection{Neumann boundary conditions}

Let us consider the self-adjoint extension corresponding to Neumann boundary conditions on the whole $\Gamma$; this is given by the direct sum $\Delta_{N}=\Delta_{\Omega_{+}}^{N} \oplus \Delta_{\Omega_{-}}^{N}$, where:

$$
\Delta_{\Omega_{ \pm}}^{N}:=\Delta \uparrow\left\{u \in H^{2}\left(\Omega_{ \pm}\right): \gamma_{1}^{ \pm} u=0\right\} .
$$

Since:

$$
\operatorname{dom}\left(\Delta_{\Omega_{+}}^{N}\right) \oplus \operatorname{dom}\left(\Delta_{\Omega_{-}}^{N}\right)=\left\{u \in H^{2}\left(\mathbb{R}^{n} \backslash \Gamma\right):\left[\gamma_{1}\right] u=\gamma_{1} u=0\right\}
$$

with the parametrization introduced in Corollary 3.5, this corresponds to the choice $\Pi(\phi \oplus \varphi):=$ $0 \oplus \varphi$, and $B_{\Theta}=0$. From (31), it follows that:

$$
\Delta_{N} u=\Delta u-\left[\gamma_{0}\right] u \nu \cdot \nabla \delta_{\Gamma}
$$

and, denoting with $Q_{z}^{ \pm}$the Neumann-to-Dirichlet operators for $\Omega_{ \pm}$respectively, the relation $\left(\gamma_{1} D L_{z}\right)^{-1}=Q_{z}^{+}-Q_{z}^{-}$, (see e.g. [1, equation (5.7)]) yields, for $z \in \mathbb{C} \backslash \mathbb{R}_{-}$,

$$
\left(-\Delta_{N}+z\right)^{-1}=(-\Delta+z)^{-1}+D L_{z}\left(Q_{z}^{+}-Q_{z}^{-}\right) \gamma_{1}(-\Delta+z)^{-1} \text {. }
$$


Next, we turn to Neumann boundary conditions supported on a relatively open part $\Sigma \subset \Gamma$ with Lipschitz boundary. We denote by $\Delta_{N, \Sigma}$ the self-adjoint extension corresponding to the orthogonal projector defined by $\Pi(\phi \oplus \varphi):=0 \oplus\left(\Pi_{\Sigma} \varphi\right) \equiv 0 \oplus(\varphi \mid \Sigma)$ and to the self-adjoint operator $\Theta(\phi \oplus \varphi):=0 \oplus\left(-\Theta_{N, \Sigma} \varphi\right)$ :

$$
\begin{gathered}
\Theta_{N, \Sigma}: \operatorname{dom}\left(\Theta_{N, \Sigma}\right) \subseteq H_{\bar{\Sigma}}^{-1 / 2}(\Gamma) \rightarrow H^{1 / 2}(\Sigma), \quad \Theta_{N, \Sigma} \varphi=\left(\hat{\gamma}_{1} D L \varphi\right) \mid \Sigma \\
\operatorname{dom}\left(\Theta_{N, \Sigma}\right):=\left\{\varphi \in H_{\bar{\Sigma}}^{1 / 2}(\Gamma):\left(\hat{\gamma}_{1} D L \varphi\right) \mid \Sigma \in H^{1 / 2}(\Sigma)\right\} .
\end{gathered}
$$

By Theorem 3.1 and (31), we have:

$$
\begin{gathered}
\Delta_{N, \Sigma} u=\Delta u-\left[\hat{\gamma}_{0}\right] u \nu \cdot \nabla \delta_{\bar{\Sigma}} \\
\operatorname{dom}\left(\Delta_{N, \Sigma}\right)=\left\{u \in H^{1}\left(\mathbb{R}^{n} \backslash \bar{\Sigma}\right) \cap L_{\Delta}^{2}\left(\mathbb{R}^{n} \backslash \Gamma\right):\left[\hat{\gamma}_{0}\right] u \in \operatorname{dom}\left(\Theta_{N, \Sigma}\right),\left[\hat{\gamma}_{1}\right] u=0, \quad\left(\hat{\gamma}_{1} u\right) \mid \Sigma=0\right\} \\
\subseteq\left\{u \in H^{1}\left(\mathbb{R}^{n} \backslash \bar{\Sigma}\right) \cap L_{\Delta}^{2}\left(\mathbb{R}^{n} \backslash \Gamma\right):\left(\hat{\gamma}_{1}^{-} u\right)\left|\Sigma=\left(\hat{\gamma}_{1}^{+} u\right)\right| \Sigma=0,\left(\left[\hat{\gamma}_{1}\right] u\right) \mid \bar{\Sigma}^{c}=0\right\}
\end{gathered}
$$

and

$$
\left(-\Delta_{N, \Sigma}+z\right)^{-1}=(-\Delta+z)^{-1}-D L_{z} \Pi_{\Sigma}^{\prime}\left(R_{\Sigma} \hat{\gamma}_{1} D L_{z} \Pi_{\Sigma}^{\prime}\right)^{-1} R_{\Sigma} \gamma_{1}(-\Delta+z)^{-1}
$$

\section{References}

[1] Mantile A., Posilicano A., Sini M. Self-adjoint elliptic operators with boundary conditions on not closed hypersurfaces. Preprint arXiv:1505.07236, 2015.

[2] Posilicano A. A Krĕın-like formula for singular perturbations of self-adjoint operators and applications. $J$. Funct. Anal., 2001, 183, P. 109-147.

[3] Posilicano A. Self-adjoint extensions by additive perturbations. Ann. Sc. Norm. Super. Pisa Cl. Sci., 2003, 2(5), P. 1-20.

[4] Posilicano A. Boundary triples and Weyl functions for singular perturbations of self-adjoint operators. Methods Funct. Anal. Topology, 2004, 10, P. 57-63.

[5] Posilicano A. Self-adjoint extensions of restrictions. Oper. Matrices, 2008, 2, P. 483-506.

[6] Albeverio S., Brasche J., Röckner M. Dirichlet forms and generalized Schrödinger operators. In: Schrödinger operators, Lecture Notes in Phys., 345, P. 1-42, Springer, Berlin, 1989.

[7] Albeverio S., Fenstad J.E., et al. Perturbations of the Laplacian supported by null sets, with applications to polymer measures and quantum fields. Phys. Lett. A, 1984, 104, P. 396-400.

[8] Albeverio S., Fenstad J.E., et al. Schrödinger operators with potentials supported by null sets, in Ideas and Methods in Mathematical Analysis (Albeverio S., Fenstad J.R., Holden H., and LindstrøT. m, Eds.), Vol. II, Cambridge Univ. Press, New York/Cambridge, MA, 1991.

[9] Antoine J.-P., Gesztesy F., Shabani J. Exactly solvable models of sphere interactions in quantum mechanics. J. Phys. A, 1987, 20, P. 3687-3712.

[10] Behrndt J., Exner P., Lotoreichik V. Schrödinger operators with $\delta$-interactions supported on conical surfaces. J. Phys. A, 2014, 47(35), 355202, 16 pp.

[11] Behrndt J., Exner P., Lotoreichik V. Schrödinger operators with $\delta$ - and $\delta^{\prime}$-interactions on Lipschitz surfaces and chromatic numbers of associated partitions. Rev. Math. Phys., 2014, 26, 1450015, 43 pp.

[12] Behrndt J., Grubb G., Langer M., Lotoreichik V. Spectral asymptotics for resolvent differences of elliptic operators with $\delta$ and $\delta^{\prime}$-interactions on hypersurfaces. J. Spectr. Theory, 2015, 5, P. 697-729.

[13] Behrndt J., Langer M., Lotoreichik V. Spectral Estimates for Resolvent Differences of Self-Adjoint Elliptic Operators. Integr. Equ. Oper. Theory, 2013, 77, P. 1-37.

[14] Behrndt J., Langer M., Lotoreichik V. Schrödinger operators with $\delta$ - and $\delta^{\prime}$-potentials supported on hypersurfaces. Ann. Henri Poincaré, 2013, 14, P. 385-423.

[15] Exner P. Spectral properties of Schrödinger operators with a strongly attractive $\delta$ interaction supported by a surface. In: Proc. NSF Summer Research Conference (Mt. Holyoke 2002), Contemp. Math., Amer. Math. Providence, R.I., 2003.

[16] Exner P. Leaky quantum graphs: a review. Proc. Symp. Pure Math., 2008, 77, P. 523-564.

[17] Exner P., Praas M. On geometric perturbations of critical Schrödinger operators with a surface interaction. $J$. Math. Phys., 2009, 50, 112101. 
[18] Exner P., Ichinose I. Geometrically induced spectrum in curved leaky wires. J. Phys. A, 2001, 34, P. 14391450.

[19] Exner P., Kondej S. Curvature-induced bound states for a $\delta$ interaction supported by a curve in $\mathbb{R}^{3}$. Ann. Henri Poincaré, 2002, 3, P. 967-981.

[20] Exner P., Kondej S. Bound states due to a strong $\delta$ interaction supported by a curved surface. J. Phys. A, 2003, 36, P. 443-457.

[21] Exner P., Kondej S. Scattering by local deformations of a straight leaky wire. J. Phys. A, 2005, 38, P. 48654874.

[22] Exner P., Pankrashkin K. Strong coupling asymptotics for a singular Schrödinger operator with an interaction supported by an open arc. Comm. Partial Diff. Eq., 2014, 39, P. 193-212.

[23] Exner P., Yoshitomi K. Band gap of the Schrödinger operator with a strong $\delta$-interaction on a periodic curve. Ann. Henri Poincaré, 2001, 2, P. 1139-1158.

[24] Figari R., Teta A. Effective potential and fluctuations for a boundary value problem on a randomly perforated domain. Lett. Math. Phys., 1992, 26, P. 295-305.

[25] Figari R., Teta A. A boundary value problem of mixed type on perforated domains. Asymptotic Anal., 1993, 6, P. 271-284.

[26] Lions J.-L., Magenes E. Non-homogeneous Boundary Value Problems and Applications. Springer, New-York, 1972.

[27] McLean W. Strongly elliptic systems and boundary integral equations. Cambridge University Press, Cambrdige, 2000.

[28] Dias N.C., Posilicano A., Prata J.N. Self-adjoint, globally defined Hamiltonian operators for systems with boundaries. Commun. Pure Appl. Anal., 2011, 10, P. 1687-1706. 